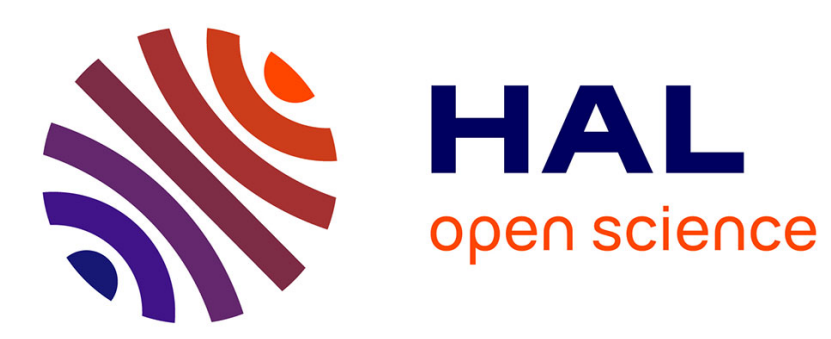

\title{
Double helix dipole design applied to magnetic resonance: A novel NMR coil
}

J. Alonso, A. Soleilhavoup, A. Wong, A. Guiga, D. Sakellariou ^

\section{To cite this version:}

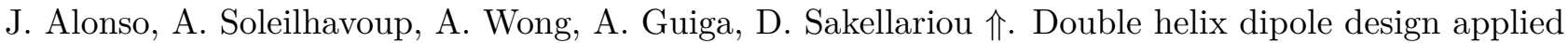
to magnetic resonance: A novel NMR coil. Journal of Magnetic Resonance, 2013, 235, pp.32-41. 10.1016/j.jmr.2013.07.004 . cea-02132455

\section{HAL Id: cea-02132455 https://hal-cea.archives-ouvertes.fr/cea-02132455}

Submitted on 17 May 2019

HAL is a multi-disciplinary open access archive for the deposit and dissemination of scientific research documents, whether they are published or not. The documents may come from teaching and research institutions in France or abroad, or from public or private research centers.
L'archive ouverte pluridisciplinaire HAL, est destinée au dépôt et à la diffusion de documents scientifiques de niveau recherche, publiés ou non, émanant des établissements d'enseignement et de recherche français ou étrangers, des laboratoires publics ou privés. 


\title{
Double helix dipole design applied to magnetic resonance: A novel NMR coil
}

\author{
J. Alonso, A. Soleilhavoup, A. Wong, A. Guiga, D. Sakellariou* \\ CEA Saclay, DSM/IRAMIS/SIS2M/LSDRM, CNRS UMR 3299, Gif-sur-Yvette 91191, France
}

\section{A R T I C L E I N F O}

\section{Article history:}

Received 7 May 2013

Revised 1 July 2013

Available online 24 July 2013

\section{Keywords:}

NMR detection

Double helix dipole

Tilted coil

NMR sensitivity

\begin{abstract}
A B S T R A C T
A new radio frequency coil design for NMR experiments is presented. The coil generates a magnetic field purely perpendicular to the longitudinal axis of the main magnet, and its sensitivity is higher than the traditional transversal resonators. This is achieved by adding the contribution of two tilted solenoid coils fed with opposite currents. The work presents the mathematical model for the new coil, numerical simulations performed to validate that model and a comparison with an equivalent saddle coil. The new design is tested experimentally in low- and high-field NMR experiments and compared with results obtained with equivalent saddle coils. The results lead to conclude that the new design provides better sensitivity than the transverse resonators commonly used in NMR.
\end{abstract}

(c) 2013 Elsevier Inc. All rights reserved.

\section{Introduction}

In NMR experiments, the nuclear magnetization of the sample under study is polarized by a strong magnetic field typically produced by a cylindrical magnet and excited by a Radio-Frequency (RF) magnetic field produced by antennas along a certain direction with respect to the main magnetic field. The electromagnetic signal induced from the excited nuclear magnetization (NMR signal) must be acquired and processed in order to complete the NMR experiment. Therefore a set of detectors or antennas (typically coils), which produce the RF magnetic field and detect the NMR signal, must be placed around the sample during the experiment. The orientation of the RF field produced by the antennas is not necessarily perpendicular to the main magnetic field. However, the larger is the perpendicular component of the RF field per unit current, the higher is the efficiency of the excitation and, by reciprocity [1], the larger is the Signal to Noise Ratio (SNR) of the NMR signal.

The solenoid provides the best performance in intensity and spatial homogeneity of the RF field when compared with other coils like Helmholtz pairs or saddle coils [2]. However, such a design does not produce a perpendicular RF field to the longitudinal axis of a cylindrical magnet unless it is placed perpendicular to it. This orientation is obviously not convenient for liquid state NMR or Magnetic Resonance Imaging (MRI) experiments, where the sample or the subject under study are usually placed with their long axis being parallel to the main magnetic field. It is also problematic

\footnotetext{
* Corresponding author.

E-mail address: dimitrios.sakellariou@cea.fr (D. Sakellariou).
}

in experiments where the sample must be spun around the magnetic field axis for improving the effective spatial uniformity. For these reasons the saddle coil is the most commonly used coil in liquid-state NMR and MRI experiments. It provides the best SNR, a reasonably good spatial homogeneity and low inductance and resistance compared with other resonators. These characteristics make it excellent for high frequency probe designs [2]. In MRI systems, other designs provide an efficient RF field perpendicular to the longitudinal axis of the main magnet. Alderman-Grant coils [3] and birdcages [4] are two standard examples of those transversal resonators. These coils could offer larger volumes of homogeneity than the saddle coil, at the expense poorer sensitivity compared with solenoids [2].

The aim of this work is to introduce a new NMR coil that produces a RF magnetic field perpendicular to its longitudinal axis with intensities higher than that produced by the resonators mentioned before. This coil is based on the magnetic dipole design [5] or double helix dipole (DHD) coil whose performance is close to that of the solenoid in terms of magnetic field intensity per unit current and spatial homogeneity.

To show these characteristics, a general overview of the DHD coil concept is presented in this work. Some examples of computed magnetic field profiles for different configurations of the new detector are also presented. Then, a formal comparison with saddle coils in terms of RF magnetic field intensity is performed. This comparison has been confirmed by experimental calibration of the $90^{\circ}$ pulse for different realizations of the new coil and different saddle coils. The work ends with a discussion about the advantages of the new resonator over conventional devices and how its performance can be further improved. 


\section{The double helix dipole coil}

A double helix dipole (DHD) magnet consists of two overlapping solenoids having tilted tight windings with respect to their longitudinal axis. They produce individually both longitudinal (solenoid) and transverse (dipole) magnetic fields at the same time. If they are fed with the same current in opposite directions, the total magnetic field will be purely transverse, since the solenoid components will vanish. The concept of the DHD magnet is presented in Fig. 1.

This concept has been applied in accelerator physics during the last decade due to two main advantages: (i) the field generated is completely transversal with respect to the axis of the coils and (ii) the value of the magnetic field generated is larger than the one produced by common transverse magnets for the same current [5]. These characteristics also fit most of the requirements for a radio-frequency NMR probe in the presence of a magnet producing a longitudinal field and justify the study presented here.

\subsection{Magnetic field of the double helix dipole coil}

Let us consider the two ideal infinitely long, tightly wound, tilted solenoids presented in Fig. 1. The first coil windings are tilted respect to the $\mathbf{z}$ axis at an angle $\alpha$ and excited with current $I$. The magnetic field generated by the coil, $\mathbf{B}_{1}$, can be decomposed into a transverse field, $\mathbf{B}_{\mathbf{y}}$, and a longitudinal field, $\mathbf{B}_{\mathbf{z}}$. If the windings are considered as single loops, the contribution to the magnetic flux density of each loop can be calculated by rotating the frame of reference at the angle $\alpha$ respect to the $\mathbf{z}$ axis [5]. Eqs. (1) and (2) define the dipole and the solenoid transfer functions (magnetic flux density per unit current T/A) of the first coil when the width of the conductor is $d$. If the winding is not tight, an effective width of the conductor, $d$, is considered. This effective width is the width of the conductor plus the pitch of the winding (see Fig. 1(b)).

$\chi_{\mathrm{y} 1}=\frac{\mu_{0}}{2 d} \cos \alpha$

$\chi_{z 1}=\frac{\mu_{0}}{d} \sin \alpha$
The second coil, which has the same dimensions as the first coil, is tilted at an angle $-\alpha$ with respect to the $\mathbf{z}$ axis and excited with the same current, $I$, of the first coil but flowing in opposite sense in axial direction. The field produced by the second coil, $\mathbf{B}_{2}$, can be also decomposed into a longitudinal field, $-\mathbf{B}_{\mathbf{z}}$, and a transverse field, $\mathbf{B}_{\mathbf{y}}$. Therefore, the solenoid transfer function of the second coil will be the opposite of the first coil, $\chi_{z 2}={ }_{-} \chi_{z 1}$, while the dipole transfer function remains constant, $\chi_{y 2}=\chi_{y 1}$. The total field produced by the superposition of the two coils is thus a transverse magnetic field with magnetic flux density, $B_{y}$, given by the following equation:

$B_{\mathrm{y}}=\frac{\mu_{0} I}{d} \cos \alpha$

In what follows, the homogeneity of the magnetic field generated by the DHD coil of Fig. 1 will be studied. It can be shown that the ideal current distribution on an infinitely long conductive cylinder, for producing the best homogeneity of a transverse magnetic field inside the cylinder, is given by Eq. (4) as has been demonstrated for birdcage coils.

$i(\theta)=I \cos \theta$

where $\theta$ is the polar angle described in Fig. 2.

It is shown in [6] that the axial current density of the DHD coil can be described by the following equation:

$J_{z}=I \frac{a \cos \theta+\frac{h}{2 \pi}}{a h d \tan \alpha}=J_{z}(\theta)+J_{z}^{s o l}$

where $I$ is the current through the coil, $a$ is the radius of the coil, $h$ is the pitch of the winding and $d$ is the wire effective diameter. This expression can be written as the sum of two terms, the first of which, $J_{z}(\theta)$, is responsible for the transverse magnetic field inside the tilted coil. The second term, $J_{z}^{\text {sol }}$, is the constant component of the axial current density responsible for the longitudinal magnetic field. Thus, the superposition of a second tilted coil with a current flowing in the opposite axial direction will cancel this constant current density, $J_{z}^{s o l}$, as was explained above. The resultant current density $2 J_{z}(\theta)$ being proportional to $\cos \theta$.

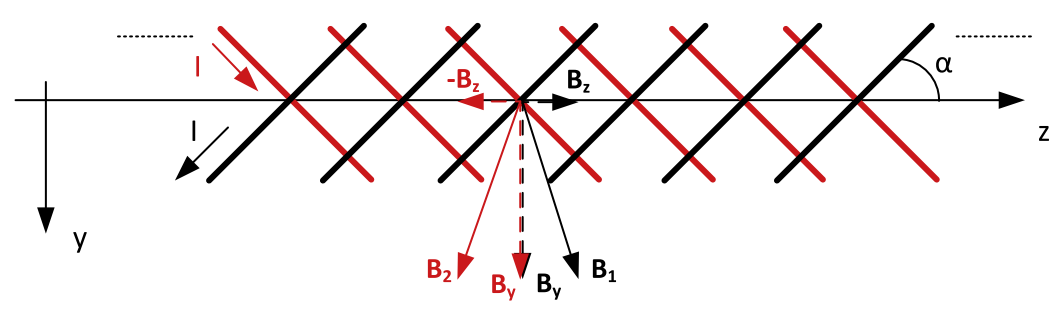

(a)

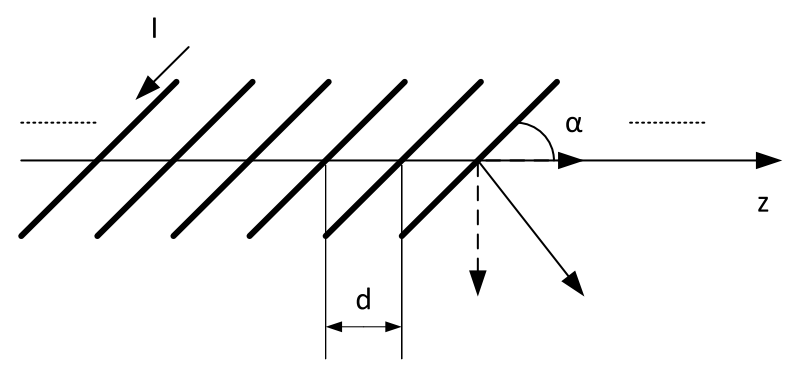

(b)

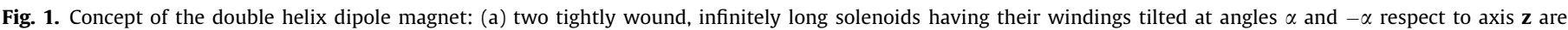

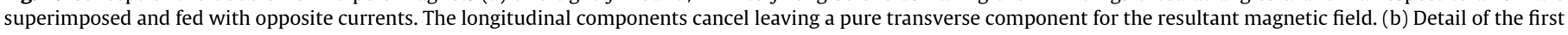
winding with the effective width of the conductor. 


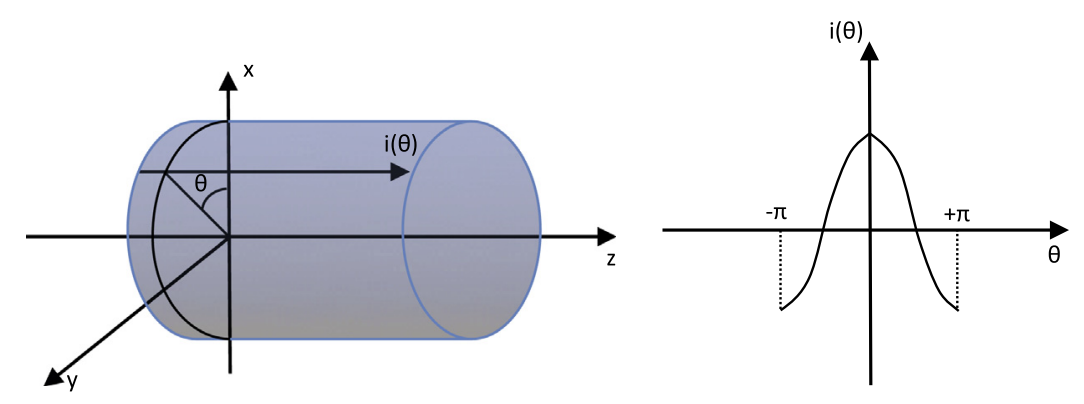

Fig. 2. A cosine-theta current distribution of the surface of cylindrical resonators produces the ideal transverse magnetic field having optimal spatial homogeneity.

This analysis shows that an infinitely long DHD helix coil has the ideal current distribution in order to produce a perfectly homogeneous transverse magnetic field. Therefore the design presented in [7] is optimized to get better sensitivity and the same homogeneity than the design presented at [8].

\subsection{Finite dipole coil: magnetic field simulations}

In the previous paragraph, the coils were considered infinitely long and wound tightly as an infinite number of single loops. This assumption is mainly valid for accelerator magnets, where the particle focusing spot is small compared with the dimensions of the coils [9] and the number of turns is very large. Also, the diameter of the coils used in accelerator magnets is large enough to consider the distance between the superimposed tilted coils small. These assumptions are however less obvious for NMR coils where the length of the coil is almost equal to the length of the sample and the distance between overlapping windings can be higher than the one tenth of the diameter of the coil. Therefore, the calculation of the magnetic flux density must consider the finite length of the coil and its practical winding, in order to give meaningful results for MR applications. A practical helical tilted winding with a pitch $h$ and radius $a$ is depicted in Fig. 3.

Biot-Savart's law predicts that the magnetic flux density at an arbitrary point, $M(x, y, z)$, for a certain current path $C$ with a constant current $I$ (see Eq. (6)).
$\boldsymbol{B}(x, y, z)=\frac{\mu_{0} I}{4 \pi} \oint \frac{\overrightarrow{d l} \times \vec{r}}{r^{2}}$

The parametric equations of the tilted coil path line with respect to the parameter $\theta$ are described in Eq. (7) [9].

$x(\theta)=a \cos \theta$

$y(\theta)=a \sin \theta$

$z(\theta)=\frac{h}{2 \pi} \theta+\frac{a \sin \theta}{\tan \alpha}=\frac{h}{2 \pi} \theta+\mathrm{a} \cdot \cot (\alpha) \sin (\theta)$

Considering the effect of the second coil placed around to the first one and centered with respect to it, the total field inside the coil is given by the following equation:

$\boldsymbol{B}(x, y, z)=\frac{\mu_{0} I}{4 \pi} \oint \frac{\overrightarrow{d l_{1}} \times \overrightarrow{r_{1}}}{r_{1}^{2}}-\frac{\mu_{0} I}{4 \pi} \oint \frac{\overrightarrow{d l_{2}} \times \overrightarrow{r_{2}}}{r_{2}^{2}}$

where the indexes 1 and 2 refer to the two coils with winding angles $\alpha$ and $-\alpha$, respectively. Notice however that the effect of the connecting wire between the two coils has not been considered into the magnetic flux density calculation of Eq. (8). Fig. 4 shows the different contour plots of the cuts in the $\mathbf{z y}$ and $\mathbf{x y}$ planes with respect to the central value of the magnetic flux density for a realistic dipole coil of a total of 24 turns, with $L=12 \mathrm{~mm}, a_{1}=2.5 \mathrm{~mm}, a_{2}=3 \mathrm{~mm}$ and $h=1 \mathrm{~mm}$, where the integrals of Eq. (8) were solved numerically (numerical integration of $\sim 80$ steps per turn).

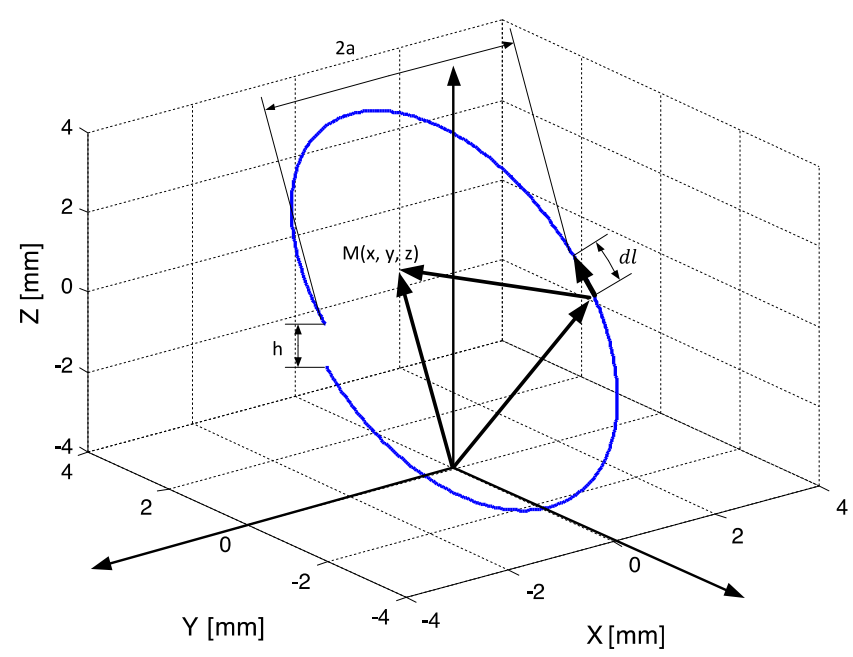

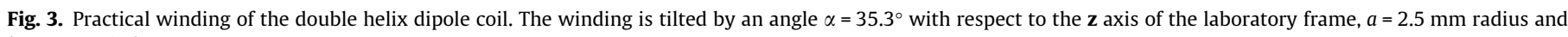
$h=1 \mathrm{~mm}$ pitch. 

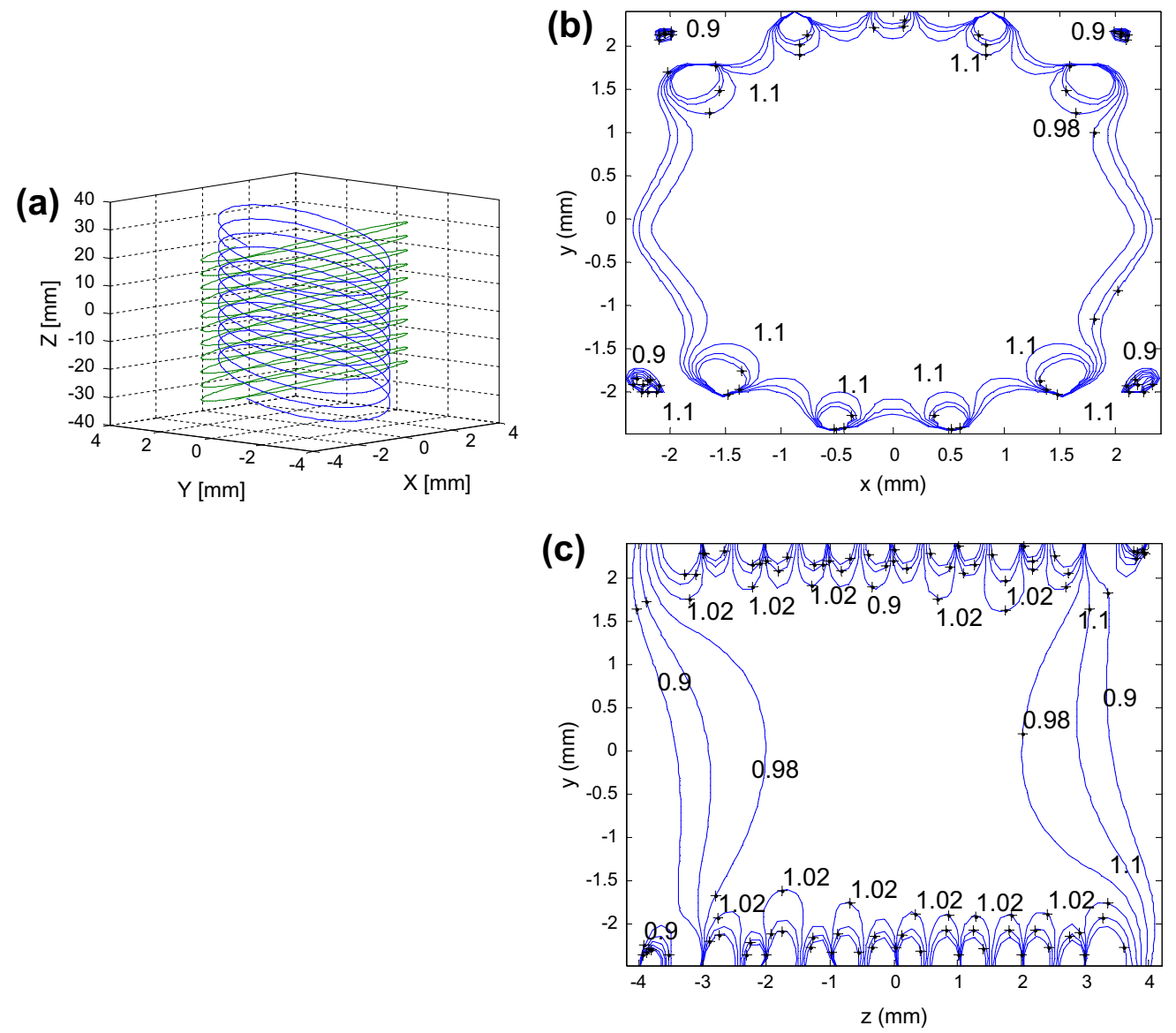

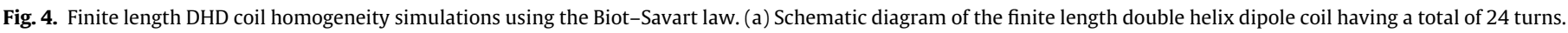

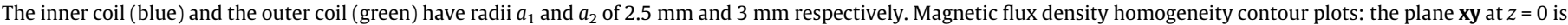

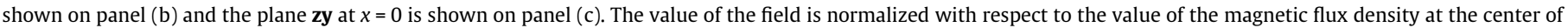
the coil. (For interpretation of the references to color in this figure legend, the reader is referred to the web version of this article.)

By numerical integration of the Biot-Savart law, the simulated value of the magnetic flux density in the $\mathbf{y}$ direction, $2 B_{1}$, is about $1.9 \mathrm{mT} / \mathrm{A}$ in the center of the coil, while the other components remain one order of magnitude smaller within the limits of the coil. The homogeneity is ensured along almost the entire volume inside the coil where the two coil windings overlap (see Fig. 4(b and c)), despite the coils have different radii $(2.5 \mathrm{~mm}$ and $3 \mathrm{~mm}$ ).

The magnetic energy stored by an inductor is defined by Eqs. (9) and (10).

$U_{m}=\frac{1}{2 \mu_{0}} \int_{V} B^{2} d V$

where the integration volume, $\mathbf{V}$, can be considered to extend only within the dimensions of the coil because the magnetic field intensity outside the coil decays to zero rapidly. The magnetic energy is related to the inductance by the following equation:

$U_{m}=\frac{1}{2} L I^{2}$

In the case of Fig. 4(a) the numerically calculated inductance is $1.06 \mu \mathrm{H}$, which is rather large for high frequency NMR applications.

Fig. 5 shows the evolution of the calculated inductance of the DHD coil with the total number of turns $N$ for different external radii. The numerical integration produces a small oscillation in the calculated values when the coil is large. However, the calculation allow to show the pseudo-linear dependence of the inductance of the DHD coil with the number of turns.

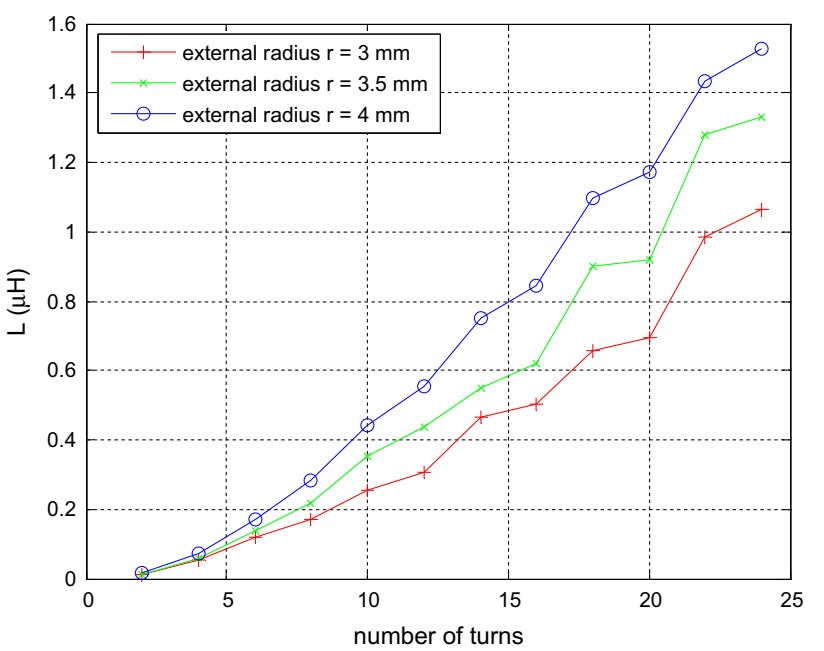

Fig. 5. Numerically calculated inductance of the DHD coil versus total number of turns for different DHD radii. (Red) $3 \mathrm{~mm}$ DHD coil inductance dependence with its number of turns. (Green) $3.5 \mathrm{~mm}$ DHD coil inductance dependence with its number of turns. (Blue) $4 \mathrm{~mm}$ DHD coil inductance dependence with its number of turns. (For interpretation of the references to color in this figure legend, the reader is referred to the web version of this article.)

These simulations show that a compromise exists between RF magnetic field intensity and working frequency. If strong RF magnetic field is required the number of turns should be increased and, 
therefore, the inductance of the coil will increase in a pseudo-linear way. This fact increases the difficulty of design a matching and tuning network for adapting the coil to the spectrometer amplifier in high frequency applications.

In order to work at high frequencies, reducing the number of turns of the coil is the obvious choice despite the loss of intensity and homogeneity of the RF field. This is what we explore with the assembly presented in Fig. 6(a), which consists of a two-turns DHD coil where the inner coil radius is $2.5 \mathrm{~mm}$, while the outer coil radius is $3 \mathrm{~mm}$. We computed the magnetic flux density and the inductance in the same way that we did for the many-turns coil. In this case, the calculated value of the magnetic flux density in the center of the coil is $0.28 \mathrm{mT} / \mathrm{A}$ (seven times wicker than the field calculated for the 22 turns DHD coil) and the calculated inductance is $13.5 \mathrm{nH}$, which is more suitable for high frequency applications.

\section{Theoretical performance of the double helix dipole coil}

\subsection{Signal to Noise Ratio of the double helix dipole coil}

The Signal to Noise Ratio equation presented in Ref. [1] can be written as:

$\Psi_{r m s} \propto V_{s} \frac{B_{1}}{I}\left(\frac{p}{l}\right)^{1 / 2}$ where $V_{s}$ is the volume of the sample, $\mathbf{B}_{1}$ is the RF magnetic field, $I$ is the current, $p$ is the perimeter of the conductor with radius $r$ and $l$ is the length of the conductor.

The loops presented in Fig. 6(a) are ellipses. The transverse component of the magnetic flux density, $\mathbf{B}_{\mathbf{t}}$, generated by one of those elliptic current loops, at its center, is given by the following equation:

$B_{t}=\frac{\mu_{0} I}{\pi b} E(k) \cos \alpha$

where $k$ is the eccentricity of the ellipse defined by the following equation:

$k=\sqrt{1-\frac{b^{2}}{a^{2}}}$

with $a$ the major semi-axis, $b$ the minor semi-axis and $E(k)$ the complete elliptical integral defined by the following equation:

$E(k)=\int_{0}^{\pi / 2} \sqrt{1-k^{2} \sin \theta} d \theta$

The derivation of Eq. (12) is developed in Appendix A.

A DHD coil having two elliptical loops tilted $\alpha$ and $-\alpha$ with respect to the $\mathbf{z}$ axis respectively and fed by opposite currents would produce in its center a magnetic flux density equal to $2 \mathbf{B}_{\mathbf{t}}$.
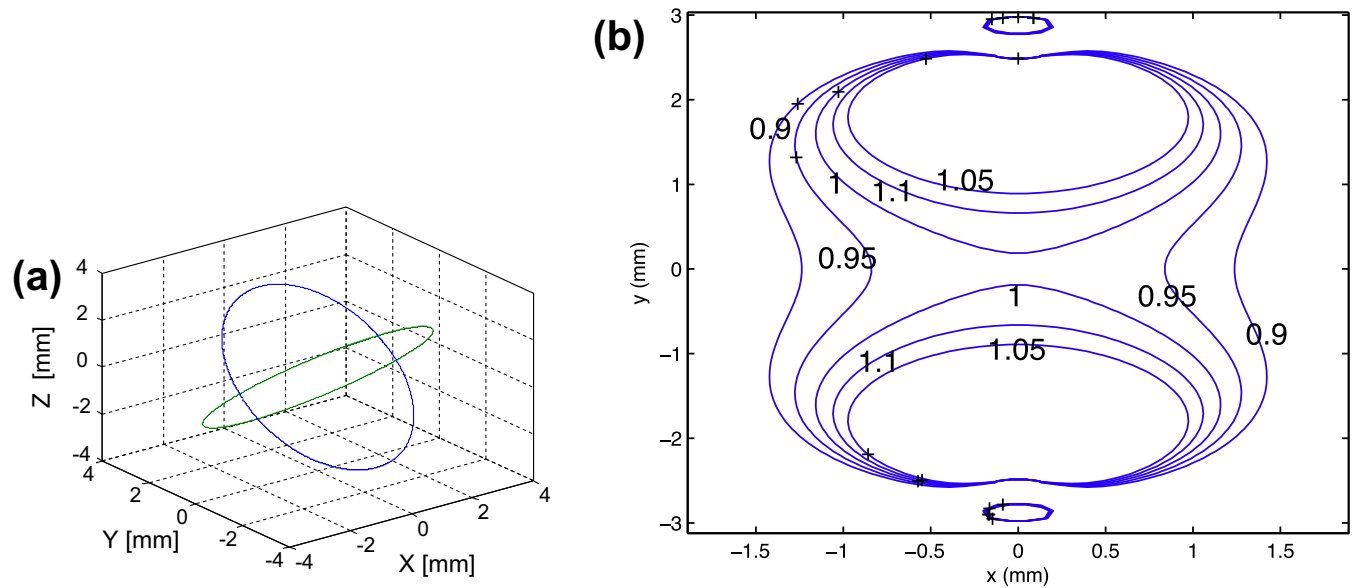

(c)

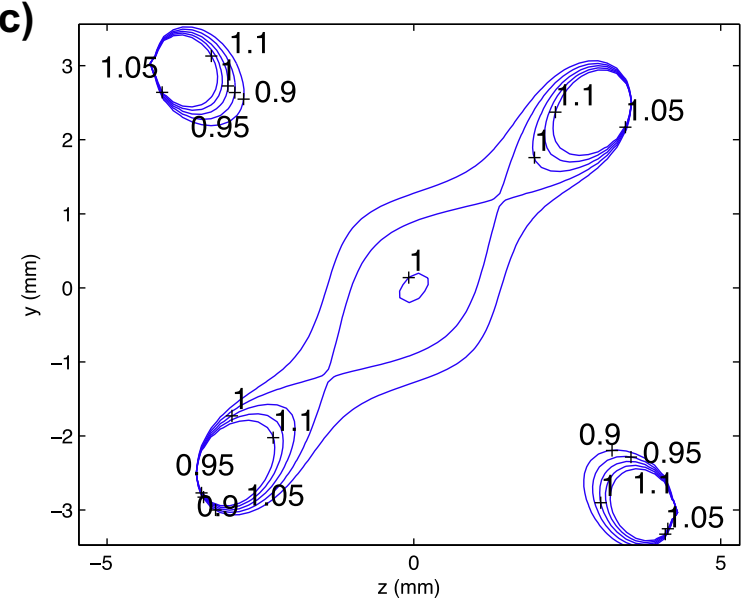

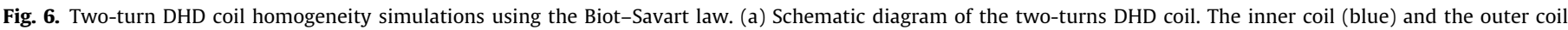

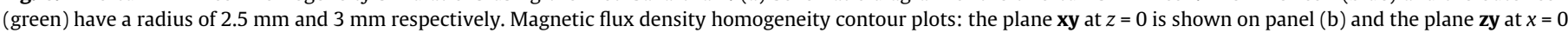

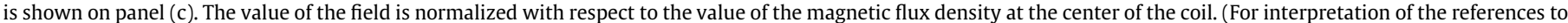
colour in this figure legend, the reader is referred to the web version of this article.) 


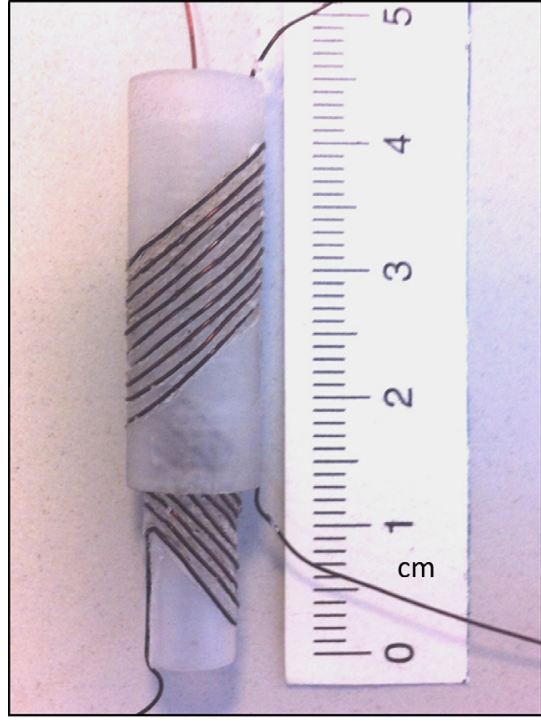

(a)

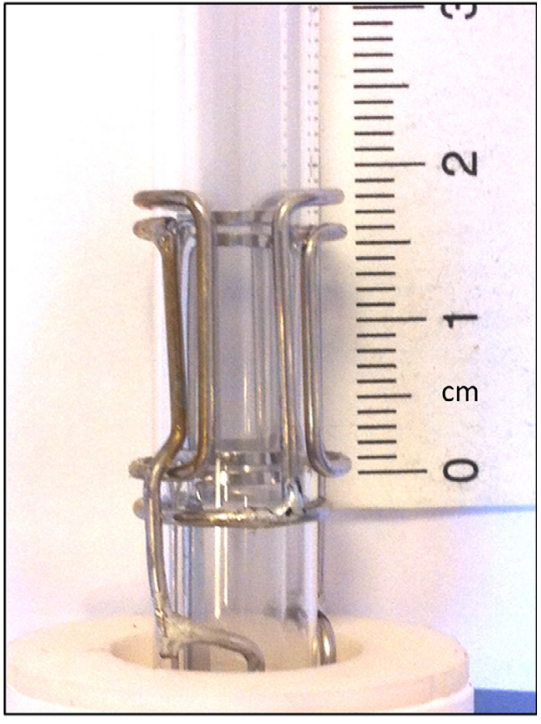

(b)

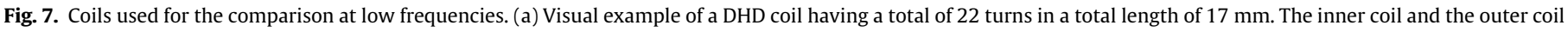
have radii of $3.5 \mathrm{~mm}$ and $4 \mathrm{~mm}$ respectively. (b) X-channel saddle coil mounted on the Bruker VSP 200 probe having a total length of $13 \mathrm{~mm}$ and a total of 4 turns.

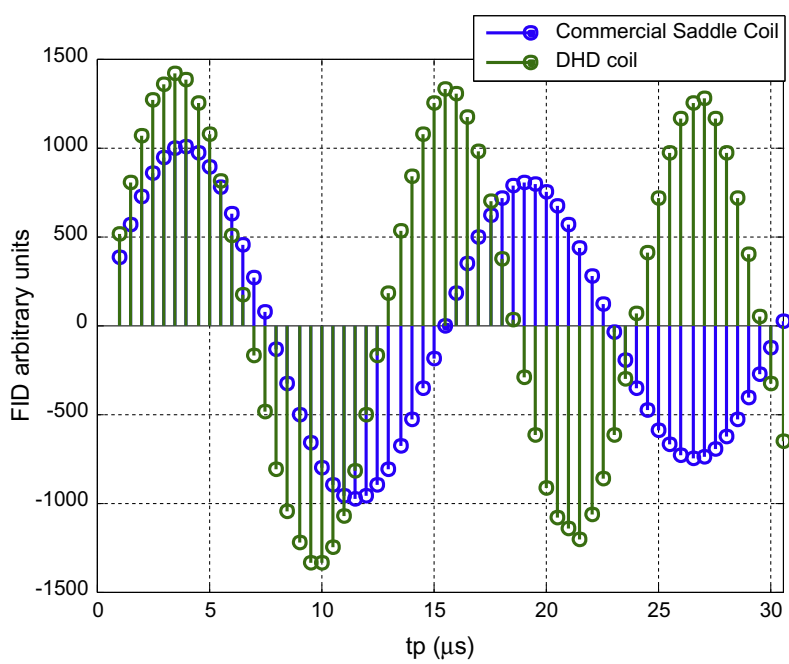

Fig. 8. FID intensity as a function of the pulse length. The probes were a DHD coil based probe (green) and a Bruker VSP 200 commercial probe (blue). The spectrometer power was $126 \mathrm{~W} @ 50 \Omega$ load. (For interpretation of the references to color in this figure legend, the reader is referred to the web version of this article.)

Therefore, the SNR of this assembly is given by Eq. (15) [1]. The derivation of this equation is presented in Appendix B:

$\Psi_{r m s}^{\text {DHD-2 turns }} \propto 0.6736 \mu_{0}\left(\frac{r}{\pi b^{3}}\right)^{1 / 2}$

A simple calculation shows that the tilting angle which optimizes this SNR is $38.57^{\circ}$.

For the two-turn DHD coil presented in the previous section, the agreement between the theoretical values and the numerical calculation of the magnetic flux density is about 93\%. The difference with simulations comes from two factors. First, the angle achieved experimentally and consider in simulations is $35.3^{\circ}$. Therefore, the transverse magnetic field is not optimized with respect to the tilting angle of the windings. Second, the approximation done by considering the two elliptical loops with the same major radius $b$. This is not true in the simulations. In simulations the radii of the coils, $a_{1}$ and $a_{2}$, are different. Therefore, the solenoid component and the component orthogonal with respect the transverse field are not zero and the value of the transverse component of the field is slightly lower.

\subsection{Comparison of the DHD coil with an equivalent saddle coil}

In order to compare the two-turn dipole coil studied before with a saddle coil optimized for high sensitivity, we calculate the general SNR for a saddle with a radius of $b$ and wound with a $2 r$ diameter wire using the following equation:

$\Psi_{\text {rms }}^{\text {saddle-2turns }} \propto 0.6286 \mu_{0}\left(\frac{r}{\pi b^{3}}\right)^{1 / 2}$

The peak SNR provided by the two-turn DHD coil is $10.5 \%$ higher than the SNR provided by the saddle coil, leading to better SNR. Using the same numerical procedure that we used for the dipole coil, the numerical value of the magnetic flux density in the center of the saddle coil is $0.017 \mathrm{mT} / \mathrm{A}$ and the calculated inductance is $12.4 \mathrm{nH}$. These simulations show that the increase on the computed SNR provided by the two-turn DHD coil is $12.5 \%$, which is in a good agreement with the theoretical value.

The last analysis concerns the homogeneity of the RF field inside the coil. Let us consider the volume of homogeneity inside each coil as a cylinder along the $\mathbf{z}$ axis where the intensity of the transversal magnetic field can vary $\pm 1 \%$. A numerical computation of the volume provides about $67 \%$ of the total volume of the DHD coil. The numerical calculation of the volume of homogeneity for the saddle coil presented above provides a $60 \%$ of the total volume of the coil. This means that the calculated volume of sample excited by the radio-frequency field inside the DHD coil is almost $10 \%$ better than the calculated volume excited by the saddle coil.

The analysis presented above suggests that the dipole coil can in principle provide better performance than the performance provided by the saddle coil, even in high frequency applications. The sensitivity of the new coil is higher than the sensitivity of the saddle coil and the spatial homogeneity is also higher. Its performance is expected to be even better in low frequency applications where 


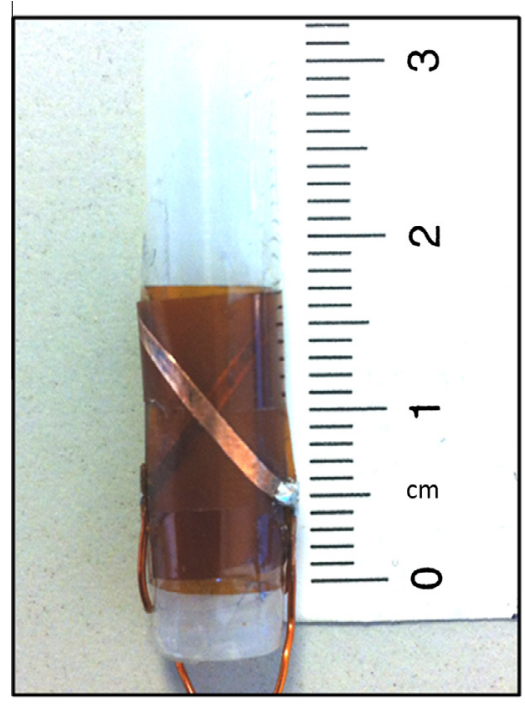

(a)

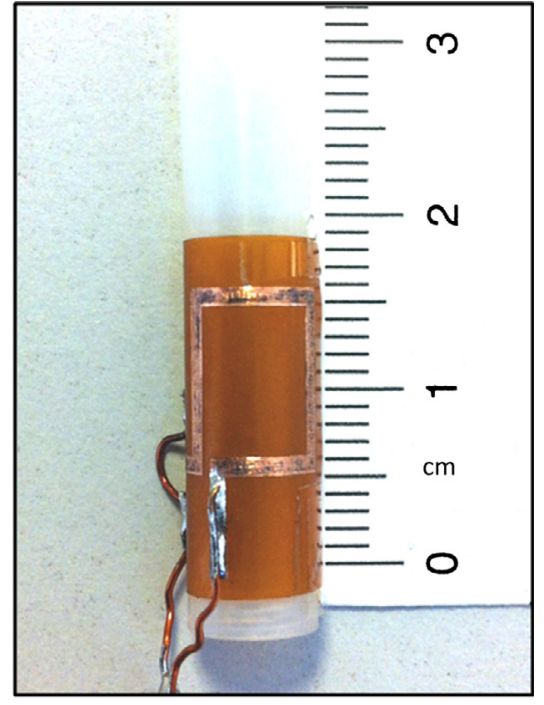

(b)

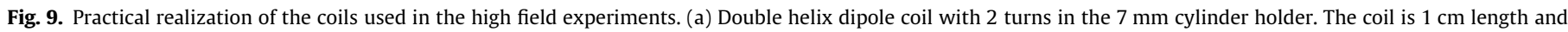
$7 \mathrm{~mm}$ radius. (b) Saddle coil with 2 turns in the $7 \mathrm{~mm}$ cylinder holder. The coil is $8.9 \mathrm{~mm}$ length and 7 mm radius, which optimizes the magnetic field inside the coil.

an increased number of turns is necessary for achieving high inductance. In the next section this improvement on the coil performance is evaluated by simple NMR experiments.

\section{NMR experiments}

Two sets of simple NMR nutation experiments were carried out in order to analyze the experimental performance of the DHD coil. The aim of the experiments was to characterize and compare the efficiency and the spatial homogeneity of the DHD coils with equivalent saddle coils at low and high magnetic fields. The results lead to conclude that the DHD coil provides better SNR in both scenarios.

\subsection{Low field NMR experiments: multiple turn dipole coil}

The low field experiments were performed in a $0.887 \mathrm{~T}\left({ }^{1} \mathrm{H}\right.$ Larmor frequency of $v_{0}=37.3 \mathrm{MHz}$ ) permanent portable magnet having a longitudinal field orientation [10]. The measured sample was $\mathrm{H}_{2} \mathrm{O}$ with $\mathrm{Cu}_{2} \mathrm{SO}_{4}$ to shorten the relaxation time, $T_{1}$, placed in a Shigemi NMR tube with a sample length restricted to $13 \mathrm{~mm}$.

The first set of experiments was carried out with the probe depicted in Fig. 7(a). The DHD coil consisted in 22 turns of $0.4 \mathrm{~mm}$ diameter copper wire wound on a mandrel with $7 \mathrm{~mm}$ diameter and $\alpha=35.5^{\circ}$. The dc resistance was $R_{\mathrm{dc}}=0.0719 \Omega$ and the inductance was $1.3 \mu \mathrm{H}$. The length of the two windings overlapping was about $15 \mathrm{~mm}$, the total length of the coil was $17 \mathrm{~mm}$ and the total length of the wire was $528 \mathrm{~mm}$. The measured Q-factor was 43 and the self resonance of the coil was placed at $105 \mathrm{MHz}$, which corresponds with a self-capacitance of $1.8 \mathrm{pF}$. After the self-resonance frequency a radiation behavior has been observed at frequencies higher than $170 \mathrm{MHz}$.

The matching network consisted of a variable tuning capacitor (1-10 pF) and a variable matching capacitor (3-23 pF). This matching network provided a good matching from $35 \mathrm{MHz}$ to $39 \mathrm{MHz}$.

The second set of low-field experiments was carried out with a commercial probe (Bruker VSP 200) depicted in Fig. 7(b) whose $\mathrm{X}$-channel was tuned to $37.3 \mathrm{MHz}$. The length of the X-channel saddle coil was $13 \mathrm{~mm}$. During the nutation experiment the samples was not spun, 4 scans of a single pulse sequence were

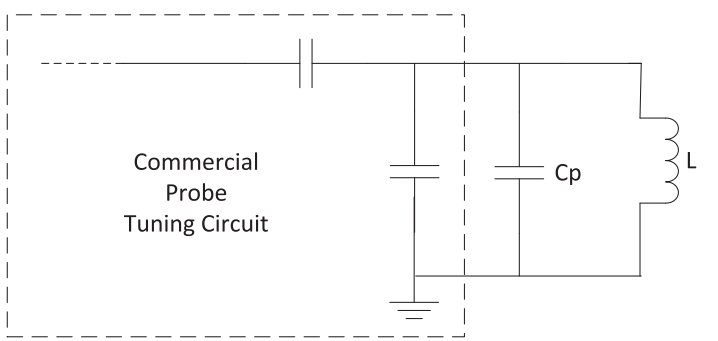

Fig. 10. Circuit model of the tuning network used in the high field experiments. $L$ is the inductance of the coils placed in the commercial probe. $C_{\mathrm{p}}$ is the pre-tuning capacitor, which set the frequency of resonance of the assembly capacitor + coil. The commercial probe is modeled as a serial-capacitance network.

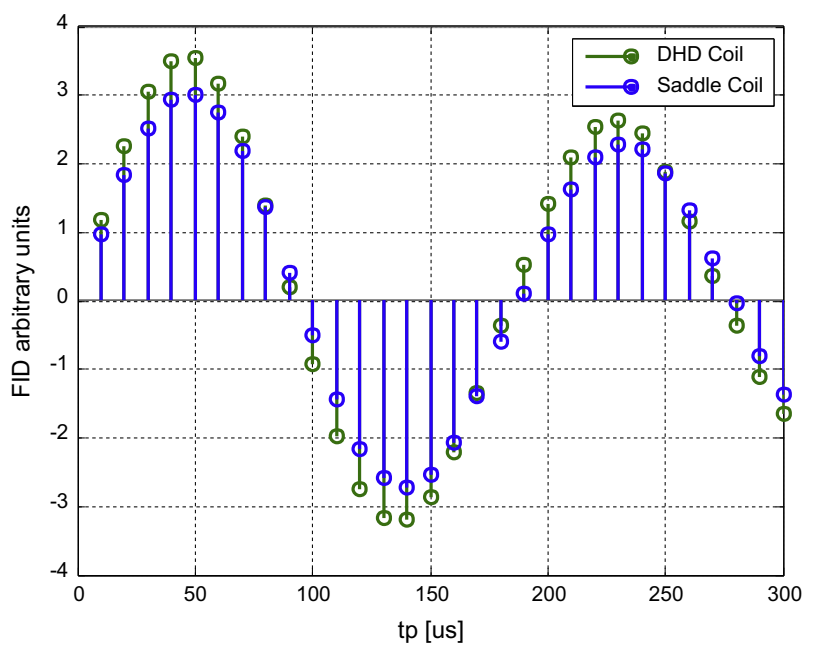

Fig. 11. FID intensity as a function of the pulse length. The probes were a DHD coil (green) and a saddle coil (blue) built in a Bruker HP WB 73A probe. Both coils were fed with $5 \mathrm{~W} @ 50 \Omega$ input power. (For interpretation of the references to color in this figure legend, the reader is referred to the web version of this article.)

recorded and no phase cycling and apodization were applied. The results are presented in Fig. 8. 
The nutation experiments showed the shorter $90^{\circ}$ pulse length for the DHD coil ( $3 \mu \mathrm{s}$ ) compared with the saddle coil commercial probe $(3.85 \mu \mathrm{s})$. This difference corresponds to a $29 \%$ increase in signal sensitivity. In accordance with the theorem of reciprocity, the FID intensity recorded from the DHD coil based probe is higher than the FID intensity recorded from the Bruker probe in a $29 \%$.

The ratio between the intensities of the Fourier transform of the FID at $450^{\circ}$ and $90^{\circ}$ shows the de-phasing of the signal due to RF in-homogeneity. Therefore, those ratios can be used to evaluate the performance of coils in terms of RF magnetic field homogeneity. The ratios are of about $93 \%$ for the dipole coil and $81 \%$ for the saddle coil. Then, the dipole coil shows an experimental increase of the RF field homogeneity of about $12 \%$.

A single echo experiment was also carried out in order to determine the ringing time of the coil. The transmitted pulse was recorded with a scope and the ringing time of the coil was measured. The dipole coil showed a ringing time about $0.8 \mu$ s during the NMR experiment. Also, measurements of the Q-factor of the DHD coil (43) were carried out, and the results suggest a coil ringing time about $1 \mu \mathrm{s}\left(\tau \sim \frac{2 Q}{\omega_{0}}\right)$ which is a good agreement with the NMR measurements.

\subsection{High field NMR experiments}

The high field experiments were carried out in Bruker UltraShielded wide-bore magnet of $11.7 \mathrm{~T}\left({ }^{1} \mathrm{H}\right.$ Larmor frequency of $v_{0}=499.6 \mathrm{MHz}$ ). The sample was $\mathrm{H}_{2} \mathrm{O}$ with $\mathrm{Cu}_{2} \mathrm{SO}_{4}$ to shorten the relaxation time, $T_{1}$, placed in a glass tube with $1 \mathrm{~cm}$ length and $7 \mathrm{~mm}$ diameter. Nutation experiments were performed at $5 \mathrm{~W}$ for two different coils.

The first set of experiments was carried out with a two-turn DHD coil. The tilted angles were $35^{\circ}$ and $-35^{\circ}$ with respect to the $\mathbf{z}$ axis. These angles are close enough to consider the coil optimized to get maximum SNR. The measured Q was about 150 and the coil is depicted in Fig. 9(a).

A saddle coil was designed to compare the performance of the dipole coil at high frequencies. The saddle coil was optimized to get the maximum magnetic field for the $7 \mathrm{~mm}$ diameter sample $[1,11]$. Therefore, the length of the saddle coil was set to $8.9 \mathrm{~mm}$. The measured $Q$ factor was about 200 . The practical realization of the coil is depicted in Fig. 9(b).

Both coils were fabricated by a lithographic process. The mask of each coil was printed over a $35 \mu \mathrm{m}$ flexible copper clad plus $50 \mu \mathrm{m}$ Kapton support. Then, the copper clad was dissolved by a corrosive solution $\left(\mathrm{FeCl}_{3}\right)$ leaving the un-protected areas free of copper.

The coils were placed in a Bruker HP WB 73A commercial NMR probe. The probe was adapted with a Teflon support for placing the designed coils at the center of the magnet. A pre-tuning capacitor, $C_{\mathrm{p}}$, of $1 \mathrm{pF}$ was placed before the commercial probe in order to tune them properly at $500 \mathrm{MHz}$. Fig. 10 shows the circuit model of the tuning network where the commercial probe is modeled as a serial-capacitance network [2].

During the nutation experiment the sample was not spun, 4 scans of a single pulse sequence were recorded and no phase cycling and no apodization were applied. The results are presented in Fig. 11.

The nutation experiments showed the $2.3 \%$ shorter $90^{\circ}$ pulse length for the DHD coil compared with the equivalent saddle coil. The difference is about $1.125 \mu$ s at $499 \mathrm{MHz}$. The ratio between FID intensities at $450^{\circ}$ and $90^{\circ}$ did not show a clear difference in-homogeneity. Both ratios were close to $80 \%$ (83\% and $78 \%$ respectively).

These results are far away from the expected values calculated in the previous section for a 2-turns DHD. These differences come from two factors. First, the tilting angle of the practical realization of the coil was $35^{\circ}$. Then the peak magnetic field is not maximized, as was shown in previous sections. Second, the alignment of the overlapped layers was performed manually. This operation is critical to obtain the calculated performance.

\section{Conclusion}

In this work the double helix dipole (DHD) design was introduced as a novel radio frequency coil for MR experiments in which the static magnetic field coincides with the direction of sample access. Although the tilted solenoid was explored before as MR radio frequency coil $[7,8]$, here, we show that the double helix dipole magnet concept can be applied in a novel way to NMR radio frequency coil technology in order to obtain better performances than previous designs. Theoretical and numerical calculations show that the improvements in SNR can be higher than 10\% when the DHD design is compared with saddle coils. 2-30\% Improvements in SNR were obtained with practical realizations of the new design.

Particular design issues have not been discussed in this work. The influence of the proximity effect due to the two layers of the DHD coil would limit the number of turns of the coil if high Q-factor in high frequency applications is required [12]. Also the self-capacitance increases with the number of turns and, therefore, the highest frequency in NMR experiments is limited.

These preliminary results prove that the opportunities of the new dipole coil are particularly promising in low field applications such as portable NMR. This technology has now reached the point where high-resolution permanent magnet systems achieve almost liquid-state resolution.

Therefore, in cases where the magnetic field is oriented along the access bore of the magnet $[10,13,14]$, this new coil could provide a high-sensitivity solution, which could be competitive with the Halbach dipole cylinder configuration. Low field applications include also DNP spectroscopy, where efficient coils for irradiation are needed while guaranteeing the sample ejection along the $\mathbf{z}$ axis. Another useful application of this coil could be the experiments where the sample is shuttled from high field to low field areas. The DHD coil can provide a good compromise for both applied magnetic fields.

The high field applications using the DHD coil might be challenging due to its high inductance. However, the preliminary increase of the magnetic field achieved by the DHD coil shown here is promising enough to study further its optimization for high frequency applications. This new coil geometry could also provide alternative solution to solid-state NMR techniques such as Magic Angle Sample or even Field Spinning, where the main magnetic field is oriented at the magic angle with respect to the longitudinal axis of the magnet and spun around the sample [15]. These directions are currently being explored in our laboratory.

\section{Acknowledgments}

The authors want to thank Dr. Jacques-Francois Jacquinot for his invaluable contributions in the electromagnetic development of the work and his experience in the practical realization of the experiments. Also, the authors want to thank Dr. D.I. Hoult, Dr. E. Fukushima and Prof. G. Bodenhausen for their comments about the manuscript. The research leading to these results has received funding from the European Research Council under the European Community's Seventh Framework Program (FP7/20072013), ERC Grant agreement 205119.

\section{Appendix A. Derivation of Eq. (12)}

Let us consider the element length $\bar{d} l$ which carries a current $I$. The vector $\bar{r}$ points to the observation point, $M$, where the magnetic 
M

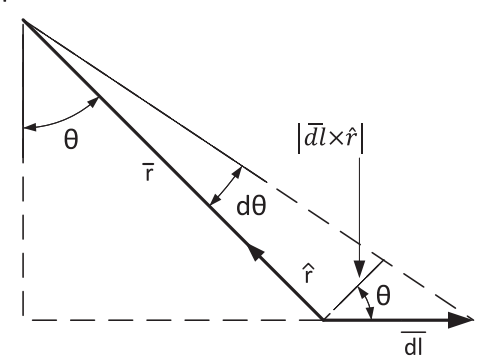

Fig. A1. Relative position of the element length with respect to the point where the magnetic field has to be calculated, $M$. The element length is denoted with $\bar{d} l$, vector $\bar{r}$ is the position vector of the element length with respect to $M$, vector $\bar{r}$ is the unit vector of $\bar{r}$ and $\theta$ is the polar angle.

field has to be calculated. The infinitesimal field contribution of this element at $M$ is given by the Bio-Savart law, described in the following equation:

$\overline{d B}=\frac{\mu_{0} I}{4 \pi} \frac{\overline{d l} \times \hat{r}}{r^{2}}$

If a finite length wire closed loop, $C$, is considered, the magnetic flux density as a function of space is:

$\bar{B}=\frac{\mu_{0} I}{4 \pi} \oint \frac{\overline{d l} \times \hat{r}}{r^{2}}$

Let us consider now the point M placed in the plane defined by the wire loop $C$. Due to the vector relations depicted in Fig. A1, the infinitesimal change in $\theta$ can be described by the following equation:

$|\bar{d} l \times \hat{r}|=r d \theta$

Therefore, the magnitude of the magnetic flux density at point $M$ is:

$B=\frac{\mu_{0} I}{4 \pi} \oint \frac{d \theta}{r}$

Let us consider the wire closed loop $C$ as a elliptic wire which center is placed at $M$ and has $a$ as major semi-axis and $b$ as minor semi-axis (see Fig. A2).

The magnitude of the magnetic flux density can be written as follows:

$B=\frac{\mu_{0} I}{\pi a b} \int_{0}^{\pi / 2} \sqrt{a^{2} \cos ^{2} \theta+b^{2} \sin ^{2} \theta} d \theta$

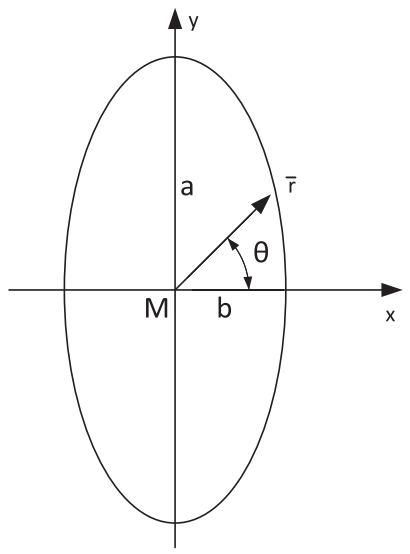

Fig. A2. Elliptic wire closed loop centered at $M$, with $a$ as major semi-axis and $b$ as minor semi-axis. Vector $\bar{r}$ denotes the position of the element length with respect to the point $\mathrm{M}$.
Eq. (A5) can be re-written as a function of the eccentricity, $k=\sqrt{1-b^{2} / a^{2}}$.

$B=\frac{\mu_{0} I}{\pi b} \int_{0}^{\pi / 2} \sqrt{1-k^{2} \sin ^{2} \theta} d \theta$

If the complete elliptical integral of the second kind is given by the following equation:

$E(k)=\int_{0}^{\pi / 2} \sqrt{1-k^{2} \sin ^{2} \theta} d \theta$

The magnitude of the magnetic flux density at the point $M$ is:

$B=\frac{\mu_{0} I}{\pi b} E(k)$

In order to derive Eq. (12) in the main corpse of the article, the wire closed loop tilted with respect of the $\mathbf{z}$-axis has to be considered. Fig. A3 depicts this arrangement of the elliptical wire closed loop. According with this figure, the component of the magnetic flux density oriented along $\mathbf{y}$-axis at the center of the loop is:

$B=\frac{\mu_{0} I}{\pi b} E(k) \cos \alpha$

\section{Appendix B. Derivation of Eq. (15)}

Let us consider the elliptical wire closed loop depicted in Fig. B1. It is mounted on a mandrel with radius $b$. The intensity of the transverse component of the magnetic flux density is given by the following equation:

$B=\frac{\mu_{0} I}{\pi b} E(k) \cos \alpha$

where $k=\sqrt{1-b^{2} / a^{2}}$, $a$ the major semi-axis, $b$ the minor semi-axis an a can be expressed as $a=\frac{b}{\sin \alpha}$.

The Signal to Noise Ratio in a Nuclear Magnetic Resonance experiment is given by Eq. (B2) [1].

$\Psi_{r m s} \propto \frac{B_{1}}{I}\left(\frac{p}{l}\right)^{1 / 2}$

Let us consider know two elliptical wire closed loops, tilted angles $\alpha$ and $-\alpha$ with respect to the $\mathbf{z}$-axis. We have called this assembly 2-turns DHD coil in the main corps of the article. If the length of the ellipse is given by $l=4 a E(k)$, the SNR of this assembly can be written as follows:

$\Psi_{r m s} \propto \frac{2 \mu_{0}}{\pi b} E(k) \cos \alpha \sqrt{\frac{2 \pi r}{8 a E(k)}}$

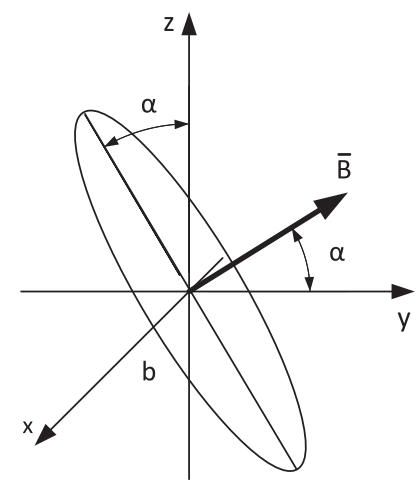

Fig. A3. Elliptic wire closed loop centered at $M$ and tilted and angle $\alpha$ with respect to the $\mathbf{z}$-axis. 


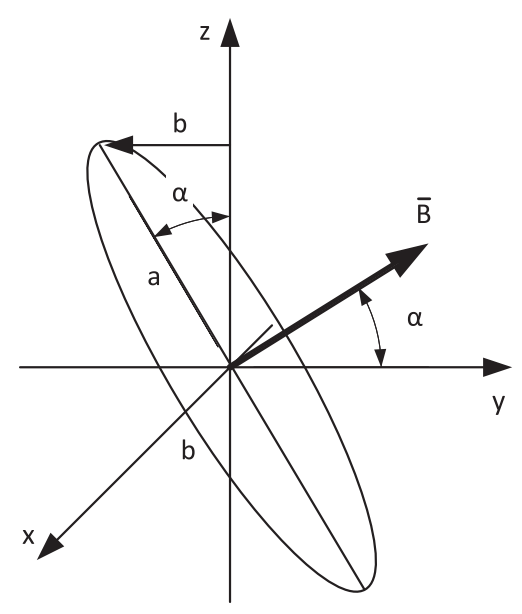

Fig. B1. Elliptic wire closed loop centered at $M$ and tilted and angle $\alpha$ with respect to the $\mathbf{z}$-axis.

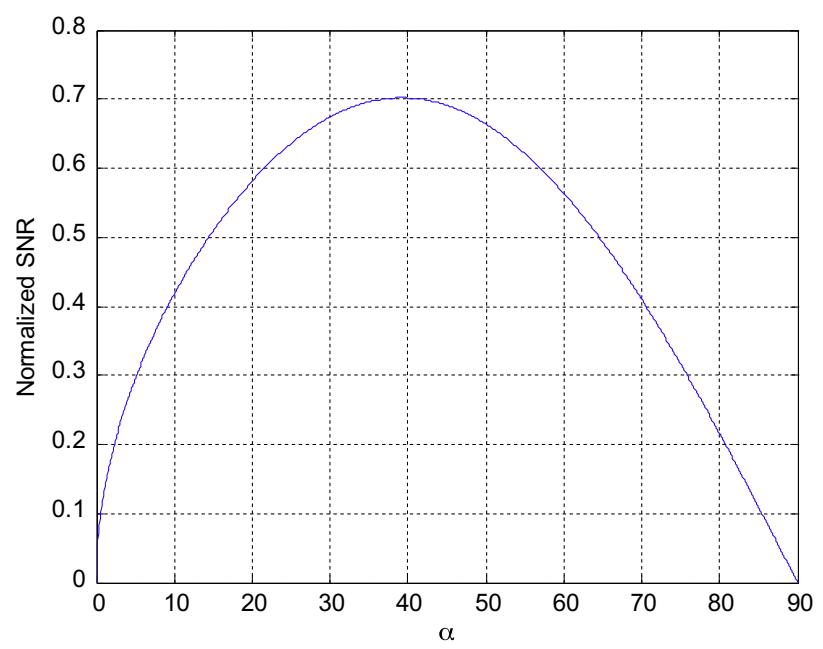

Fig. B2. Evolution of the angular part of Eq. (B4) with $\alpha$.

If $a=\frac{b}{\sin \alpha}$, the SNR of the 2-turns DHD coil is defined by the following equation:
$\Psi_{r m s} \propto \frac{\mu_{0}}{b} \sqrt{\frac{r}{\pi b}} \cos \alpha \sqrt{E(k) \sin \alpha}$

The definition of the eccentricity allows to put the complete elliptical integral of the second kind as a function of $\cos (\alpha)$, Therefore, the SNR can be maximize in terms of $\alpha$. Fig. B2 shows the evolution of the normalized SNR as a function of the angle $\alpha$.

The maximum of the SNR occurs at $\alpha=38.57^{\circ}$. At this value Eq. (B4) takes the following value:

$\Psi_{r m s} \propto 0.6736 \frac{\mu_{0}}{b} \sqrt{\frac{r}{\pi b}}$

\section{References}

[1] D. Hoult, R. Richards, The signal-to-noise ratio of the nuclear magnetic resonance experiment, J. Magn. Reson. 24 (1976) 71-85.

[2] J. Mispelter, M. Lupu, A. Briguet, NMR Probeheads for Biophysical and Biomedical Experiments, Imperial College Press, 2006

[3] D.W. Alderman, D.M. Grant, Efficient decoupler coil design which reduces heating in conductive samples in superconducting spectrometers, J. Magn. Reson. 36 (1979) 447-451.

[4] C. Hayes, W. Edelstein, J. Schenck, O. Muller, M. Eash, An efficient, highly homogeneous radiofrequency coil for whole-body NMR imaging at $1.5 \mathrm{~T}, \mathrm{~J}$. Magn. Reson. 63 (1985) 622-628.

[5] C. Goodzeit, M. Ball, R. Meinke, The double-helix dipole. A novel approach to accelerator magnet design, IEEE Trans. Appl. Supercond. 13 (2003) 1365-1368.

[6] A. Akhmeteli, A. Gavrilin, Superconducting and resistive tilted coil magnets for generation of high and uniform transverse magnetic field, IEEE Trans. Appl. Supercond. 15 (2005) 1439-1443.

[7] Y. Sun, G.E. Maciel, The tilted coil for NMR experiments, J. Magn. Reson., Ser. A 105 (1993) 145-150.

[8] E. Jeong, D. Kim, S. Lee, J. Sus, Y. Kwon, A solenoid-like coil producing transverse RF fields for MR imaging, J. Magn. Reson. 127 (1) (1997) 73-82.

[9] S. Farinon, P. Fabbricatore, Refined modeling of superconducting double helical coils using finite element analyses, Supercond. Sci. Technol. 25 (2012) 10

[10] C. Hugon, F. D’Amico, G. Aubert, D. Sakellariou, Design of arbitrarily homogeneous permanent magnet systems for NMR and MRI: theory and experimental developments of a simple portable magnets, J. Magn. Reson. 205 (2010) 75-85.

[11] D.I. Hoult, The NMR receiver: a description and analysis of design, Prog. NMR Spectrosc. 12 (1978) 41-77.

[12] I.M. Savukov, S.J. Seltzer, M.V. Romalis, Detection of NMR signals with a radiofrequency atomic magnetometer, J. Magn. Reson. 185 (2007) 214-220.

[13] H. Zijlstra, Permanent magnet systems for NMR tomography, J. Philips Res. 40 (1985) 259-288.

[14] K. Halbach, Design of permanent multipole magnets with oriented rare earth cobalt materials, Nucl. Instrum. Methods 169 (1980) 1-10.

[15] D. Sakellariou, C. Hugon, A. Guiga, G. Aubert, S. Cazaux, P. Hardy, Permanent magnet assembly producing a strong tilted homogeneous magnetic field: towards magic angle field spinning NMR and MRI, Magn. Reson. Chem. 8 (2010) 903-908. 